\title{
Análise de Mecanismos de Serverless Computing em Ambientes de Nuvens Computacionais
}

\author{
Matheus N. da Silva, Marcus Carvalho \\ Departamento de Ciências Exatas - Universidade Federal da Paraíba (UFPB), Campus \\ IV - Av. Santa Elizabeth, S/N, Centro - CEP 58297-000 - Rio Tinto - PB - Brazil \\ \{matheus.nicolas, marcuswac @dcx.ufpb.br
}

\begin{abstract}
The serverless computing model is trending nowadays, because of its easy adoption and possibility of reducing costs. However, as users are not in charge of managing servers, they may face performance issues related to coldstarts, when the provider disables an idle application and new requests have to wait until it is deployed in a server and enabled again. The objective of this work is to analyze coldstarts in serverless computing, aiming to understand the time an application stays idle until the provider disables it, the overhead implied by coldstarts on response times and the impact of application's memory size on these metrics.

Resumo. O modelo de serverless computing tem se tornado tendência nos últimos anos, devido à facilidade de adoção e possibilidade de redução de custos. Porém, como usuários não têm controle sobre os servidores, eles podem ter problemas de desempenho relacionados a coldstarts, quando o provedor torna inativa uma aplicação ociosa e novas requisições precisam esperar até que ela seja implantada em um servidor e se torne ativa novamente. $O$ objetivo deste trabalho é analisar o coldstart em serverless computing, buscando entender o tempo de ociosidade de uma aplicação até o provedor torná-la inativa, o overhead imposto pelo coldstart no tempo de resposta e se a quantidade de memória alocada para a aplicação afeta essas métricas.
\end{abstract}

\section{Introdução}

A computação em nuvem permite a seus usuários reduzir despesas na aquisição de hardware e em sua manutenção, investindo por outro lado em serviços terceirizados pelos provedores. Inicialmente, ainda cabia ao usuário da nuvem realizar tarefas operacionais como: criar instâncias (servidores) e determinar sua capacidade (e.g. CPU, memória e disco); implantar a aplicação nos servidores; e decidir quando e quantas instâncias estarão em execução com base na demanda da aplicação [Savage 2018].

O serverless computing surgiu como alternativa a este modelo tradicional, no qual os usuários não precisam gerenciar os servidores que rodam suas aplicações. Neste novo modelo, o usuário pode focar de fato na sua aplicação, deixando para o provedor a implantação da aplicação em instâncias, escalabilidade e tolerância a falhas. O usuário paga apenas pelo uso de suas aplicações com base na demanda e não mais pelo tempo em que os servidores estiveram rodando [Fowler 2012].

Um dos principais modelos associados ao serverless é o FaaS (ou função como serviço, do inglês Function as a Service). Neste modelo, o usuário carrega o código da função no provedor, que é responsável por toda a gerência de servidores, implantação da função em um ambiente de execução e provisionamento automático de recursos para atender à demanda. A execução da função é acionada por eventos definidos pelo usuário no provedor - por exemplo, uma requisição HTTP que chega em um certo caminho pode acionar uma função que faz o seu processamento [Fowler 2012]. Provedores de FaaS da atualidade incluem Amazon AWS Lambda' e Google Cloud Functions ${ }^{2}$, que dão

https://aws.amazon.com/pt/lambda/ 
suporte a funções implementadas em linguagens de programação e ambientes de execução como: Node.js, Python, Go, Java, etc.

Um dos diferenciais do modelo serverless é que, para reduzir custos, o provedor pode desativar aplicações após um tempo de ociosidade (i.e. em períodos sem demanda). Porém, para processar requisições que chegam para uma aplicação inativa, o provedor precisa antes implantar esta aplicação em um servidor e iniciá-la em um ambiente de execução. Este tempo de inicialização de aplicações inativas em um ambiente serverless é chamado de coldstart, que pode impactar o desempenho das aplicações [Baldini et al 2016].

O objetivo geral deste trabalho é investigar o coldstart em um ambiente de serverless computing, analisando o seu impacto no tempo de resposta das aplicações e os fatores que podem afetar a sua frequência e duração. Para isto, foram realizados experimentos de medição em um provedor de $\mathrm{FaaS}$, usando funções e cargas sintéticas. Como objetivos específicos, buscou-se responder às seguintes perguntas de pesquisa:

- Com quanto tempo de ociosidade uma função é desativada pelo provedor, tendo como consequência o coldstart em requisições que vierem a seguir?

- Qual o tempo adicional (overhead) que o coldstart impõe no tempo de resposta das requisições, comparado a requisições que não passam por coldstart?

- Qual o impacto da quantidade de memória alocada para a função no overhead do coldstart e no intervalo de ociosidade para que ocorra um coldstart.

O restante do artigo está estruturado da seguinte forma: a seção 2 apresenta os trabalhos relacionados; a seção 3 apresenta a metodologia da avaliação; a seção 4 apresenta os resultados; e a seção 5 discute as conclusões e trabalhos futuros.

\section{Trabalhos relacionados}

Baldini et al. (2016) faz um levantamento das tendências e problemas em aberto na área de serverless computing. Um dos desafios apontados é exatamente o fenômeno do coldstart, pois ao mesmo tempo que pode-se reduzir custos escalando uma função para zero servidores, desativando-a, pode-se também piorar seu desempenho por causa do coldstart. Por isso, seu trabalho aponta como crítico o estudo de técnicas para minimizar o coldstart, ao mesmo tempo que aplicações ociosas ainda possam ser desativadas. Apesar da relevância do tema, encontramos poucas publicações acadêmicas que realizam análises voltadas ao coldstart durante nossa revisão da literatura. Desta forma, o trabalho corrente visa preencher um pouco esta lacuna, contribuindo com uma avaliação de desempenho de coldstarts em um ambiente de serverless computing.

Os trabalhos encontrados mais relacionados foram os de Cui (2017a) e Cui (2017b), que apesar de não terem sido publicações acadêmicas, apresentam uma metodologia clara e resultados bem descritos. Em seu primeiro estudo, Cui (2017a) propõe uma metodologia de detecção de coldstarts e analisa um provedor de FaaS (AWS Lambda) quanto tempo uma função fica ociosa até que o provedor torne-a inativa, resultando em seguida em um coldstart. Este estudo concluiu que o AWS Lambda tornou uma função inativa após aproximadamente 40 a 60 minutos de ociosidade, mas que eventualmente podiam ocorrer coldstarts antes desse período de ociosidade, para liberar recursos. Também observou-se que funções com mais memória alocada tendem a ser desativadas com um tempo menor de ociosidade. Com base nesta análise inicial, nosso trabalho buscou reproduzir os experimentos de Cui (2017a) para medir quando os coldstarts ocorrem e, além disso, expandir a análise para medir o overhead imposto pelo coldstart no tempo de resposta das funções, dependendo da quantidade de memória alocada para ela. 
Em outro estudo, Cui (2017b) mediu como a linguagem de programação, o tamanho do código e a quantodade de memória alocada afetam o coldstart. Em seus resultados, observou-se que: linguagens de programação diferentes podem apresentar diferenças significativas no overhead de coldstarts, sendo os coldstarts para Python e NodeJS bem menores que para Java e C\#, quanto mais memória alocada para a função, menor a duração do coldstart; quanto maior o código, menor o coldstart. O nosso trabalho buscou fazer uma análise mais aprofundada da duração e frequência de coldstarts para diferentes tamanhos de memória, focando no framework NodeJS. Ao contrário do trabalho de Cui (2017b), observamos que a relação do tempo do coldstart com o tamanho da memória alocada não é linear, o que será discutido nos resultados.

\section{Metodologia}

Neste trabalho, foram realizados experimentos de medição para analisar a frequência e duração de coldstarts em um ambiente de serverless computing. $\mathrm{O}$ experimento foi baseado na metodologia usada por Cui (2017a) para criação de funções, medição de desempenho e geração de carga.

O ambiente de FaaS do AWS Lambda foi usado para a medição de desempenho e análise de coldstarts. Uma função lambda chamada system-under-test foi criada no ambiente de execução NodeJS, sendo configurada para executar ao chegarem requisições HTTP em um caminho específico. Esta função informa como retorno se a requisição sendo executada está se deparando ou não com um coldstart da seguinte forma: em seu primeiro acesso, a função sendo executada armazena uma flag em memória indicando que já foi acessada; se um acesso posterior à função não observar tal flag ativada, é porque a função passou por um coldstart e teve que ser iniciada novamente, perdendo seu estado prévio [Cui 2017a]. Com o intuito de avaliar o impacto da alocação de memória no coldstart, foram executados diferentes cenários de alocação de memória para a função system-under-test, com os seguintes valores: $128 \mathrm{MB}$, $256 \mathrm{MB}, 512 \mathrm{MB}, 1024 \mathrm{MB}$ e $2048 \mathrm{MB}$.

A geração de carga e medição de desempenho foi realizada utilizando o serviço AWS Step Functions ${ }^{3}$, que possibilita a criação de um fluxo de execução de funções e a gravação de medições em serviços de monitoramento. A Figura 1, demonstra as funções criadas e o fluxo de execução para a geração de carga periódica dos experimentos.

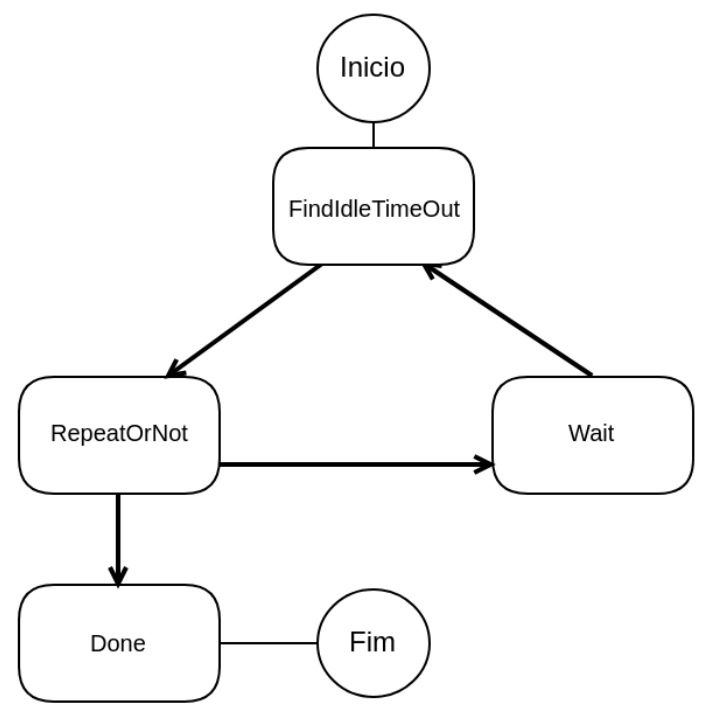

Figura 1. Fluxo de execução de funções de geração de carga dos experimentos.

\footnotetext{
${ }^{3}$ https://aws.amazon.com/pt/step-functions/
} 
Durante a execução de um experimento, o fluxo de execução mantém uma máquina de estados com os três atributos abaixo:

- target: função alvo a ser chamada pelo gerador de carga e para a qual o desempenho será medido;

- coldstarts: contador da quantidade de coldstarts consecutivos encontrados;

- wait: intervalo de tempo entre requisições submetidas pelo gerador de carga.

A função FindIdleTimeout é responsável por gerar a carga e medir o tempo de resposta, enviando requisições periodicamente à função system-under-test informada no parâmetro target. Cada chamada à função system-under-test retorna o status do coldstart para aquela chamada: se houve colstart, o parâmetro coldstarts do experimento é incrementado. Em seguida, o fluxo do experimento passa para a função RepeatOrNot.

A função RepeatOrNot verifica se o experimento chegou ao fim, ou se ainda serão necessários mais testes. Além disso, ela pode ajustar o parâmetro wait para determinar o tempo de espera até a próxima requisição ser gerada. Um dos objetivos do experimento é saber após quanto tempo de ociosidade o provedor torna a função inativa - ou seja, quando os coldstarts começam a acontecer sistematicamente. Nos nossos experimentos, o parâmetro wait é iniciado com o valor de 10 minutos, sendo incrementado ao longo do experimento até que seja encontrado o tempo de ociosidade que causa coldstarts de forma sistemática. Como coldstarts inesperados podem acontecer mesmo com pouco tempo de ociosidade, considera-se que o tempo de ociosidade sistemático para tornar a função inativa é encontrado quando 10 coldstarts consecutivos são observados. Desta forma, se a função RepeatOrNot verificar que a variável coldstarts chegou a 10, o fluxo do experimento segue para o estado Done para ser finalizado. Caso contrário, se não houve coldstart na última requisição, tenta-se um tempo de ociosidade maior incrementando o atributo wait em 1 minuto e zerando o contador de coldstarts para este novo período. No fim, o fluxo passa para a função Wait.

A função Wait representa um simples estado de espera, onde a variável wait é observada para saber quantos minutos o fluxo de execução do experimento ficará aguardando naquele estado, até voltar novamente à função FindIdleTimeout para gerar mais uma requisição e continuar o ciclo.

Esse fluxo de execução do experimento foi rodado uma vez para cada cenário de alocação de memória, um cenário de cada vez. A execução de todos os cenários durou aproximadamente um dia para executar por completo, devido as várias rodadas necessárias para encontrar o tempo de ociosidade (wait) que cause 10 coldstarts consecutivos.

As métricas avaliadas no experimento foram:

- tempo máximo ocioso: tempo máximo que uma função permanece ociosa, sem receber requisições, até ser desativada pelo provedor. Essa métrica foi calculada como o maior valor da variável wait, para o qual foram observados 10 coldstarts consecutivos.

- tempo de resposta das requisições: tempo observado pelo gerador de carga entre a submissão de uma requisição à função system-under-test e o recebimento de sua resposta. Associada a esta medição também está a identificação da requisição enviada ter ou não passado por um coldstart.

A próxima seção apresenta os resultados da avaliação, discutindo as métricas para cada cenário descrito na metodologia. 


\section{Resultados}

A Tabela 1 apresenta os resultados do tempo máximo ocioso para diferentes cenários de memória alocada para a função. Observa-se que, nos experimentos, o AWS Lambda desativou a função de forma sistemática após um tempo de ociosidade que variou de 30 a 42 minutos. O tempo máximo ocioso observado nos experimentos foram menores do que os encontrados por Cui (2017a), 1 ano antes, que reportou valores entre 48 e 63 minutos para os mesmos cenários de memória alocada. Este resultado sugere que o tempo de ociosidade para desativar uma função não é constante e que, além do tamanho da memória, ele pode depender de fatores externos como a carga do provedor. Apesar de Cui (2017a) sugerir que existe um padrão de quanto mais memória alocada, menor o tempo máximo ocioso, este fenômeno não foi observado nos nossos experimentos. $\mathrm{O}$ tempo máximo ocioso de fato variou ao mudar o total de memória alocada, mas não conseguimos observar nenhum padrão linear. Uma quantidade maior de experimentos devem ser realizados, em diferentes dias e horas, para tentar capturar apenas o efeito da alocação de memória no tempo máximo de ociosidade.

Tabela 1. Tempo máximo ocioso da função para o qual acontecem coldstarts sistemáticos, para diferentes cenários de memória alocada.

\begin{tabular}{|c|c|}
\hline Total de memória alocada (MB) & Tempo máximo ocioso (minutos) \\
\hline 128 & 30 \\
\hline 256 & 42 \\
\hline 512 & 30 \\
\hline 1024 & 30 \\
\hline 2048 & 40 \\
\hline
\end{tabular}

A Figura 2 apresenta o tempo de resposta das requisições ao variar o intervalo entre requisições (wait), em diferentes cenários de memória alocada para a função. Para cada requisição, também é identificado se a mesma passou por um coldstart (em azul) ou se não passou por coldstart (em vermelho). Nota-se que sempre há um coldstart no intervalo inicial entre requisições, de 10 minutos. Isto acontece porque a primeira requisição sempre passa por um coldstart, pois ela que ativa a função pela primeira vez. Também pode-se notar claramente que o tempo de execução com coldstart é significativamente maior do que o tempo de resposta sem coldstart, reforçando a importância de minimizar ao máximo coldstart. Também se observa que os coldstarts tendem a aparecer aproximadamente após 25 minutos de ociosidade, mas nem sempre a função é desativada de forma sistemática em um intervalo fixo. Uma hipótese é que em momentos de sobrecarga no provedor, ele pode decidir desativar funções que estão ociosas a mais de 25 minutos, dando prioridade a funções que estão ociosas a mais tempo.

A função de maior alocação de memória (2048MB) foi a que mais apresentou coldstarts antes de atingir os 10 consecutivos, havendo: 5 coldstarts com ociosidade de 26 minutos; 2 coldstarts com ociosidade de 35 minutos; e 10 coldstarts com ociosidades de 10 minutos, quando considerou-se o tempo de ociosidade sistemática. Uma outra hipótese para estes coldstarts mais frequentes em intervalos mais curtos é que o provedor pode tentar desativar primeiro funções que estão ociosas por algum tempo e que possuem mais memória alocada, para liberar mais recursos em períodos de sobrecarga no servidor. 
coldstart nao $\bullet$ sim
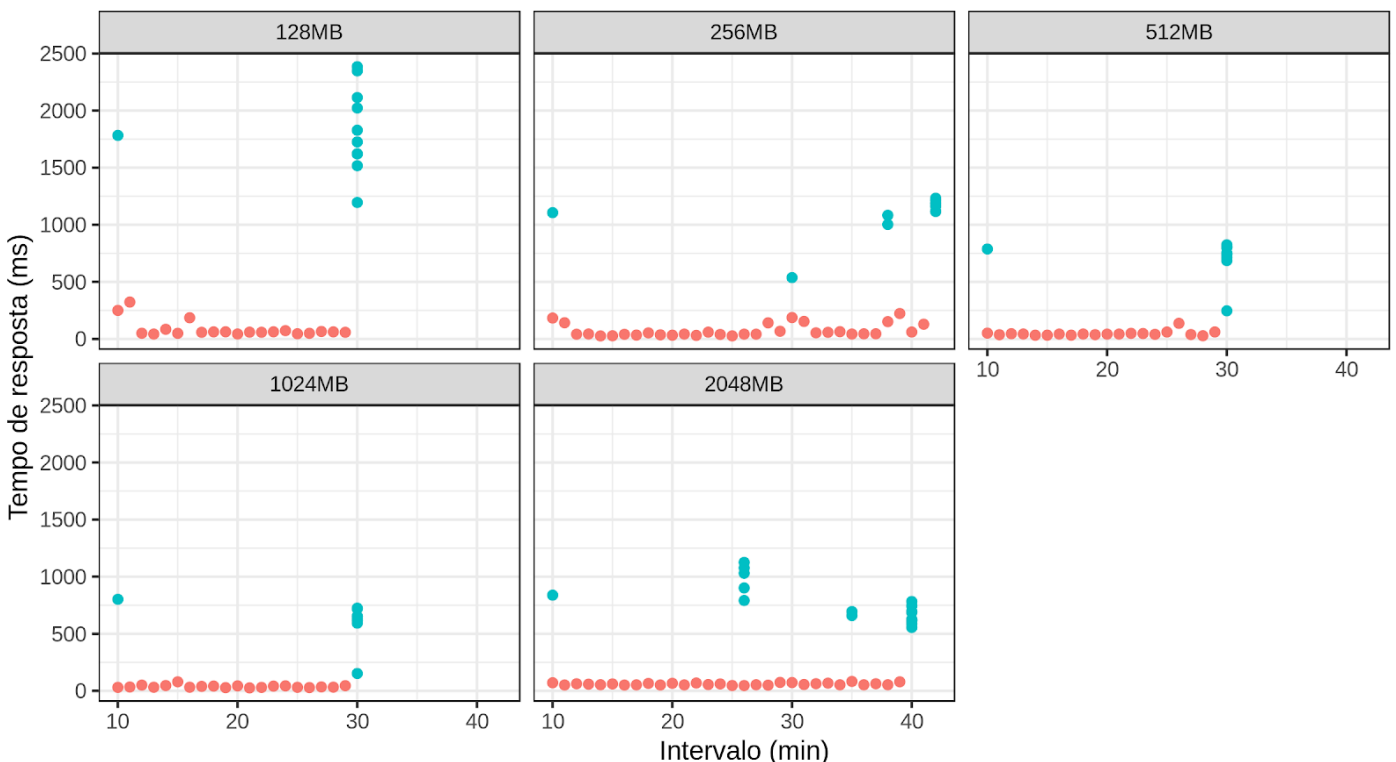

Figura 2. Tempo de resposta das requisições com e sem coldstart.

A Figura 3 apresenta o boxplot utilizado para analisar o tempo de resposta das requisições para todos os cenários. Podemos comparar as medianas e os quartis do tempo de resposta quando há ou não coldstart. Os pontos dispersos presentes na figura são chamados de outliers; são os valores discrepantes no nosso gráfico e a presença deles nos experimentos é a razão de fazermos a comparação através da mediana e não da média. Observa-se que o tempo de resposta com coldstart foi significativamente maior do que sem coldstart. A mediana do tempo de resposta sem coldstart foi de $51 \mathrm{~ms}$, enquanto com coldstart a media foi $802 \mathrm{~ms}$. Ou seja, o tempo adicional gasto com o coldstart foi de aproximadamente $751 \mathrm{~ms}$ para os cenários avaliados.

coldstart 由 nao 追 sim

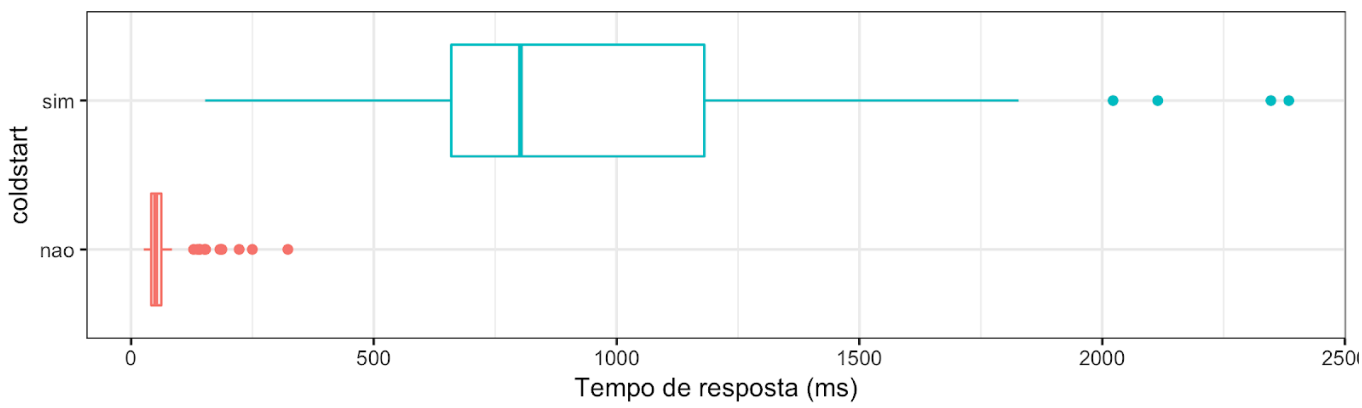

Figura 3. Boxplot do tempo de resposta total com e sem coldstart.

A Figura 4 apresenta o boxplot do tempo de resposta com e sem coldstart, agora dividindo para cada cenário de memória alocada. Observando o gráfico do tempo de execução quando ocorrem coldstarts (à direita), é possível identificar uma tendência: quanto mais memória alocada, menor é o tempo de coldstart. Este comportamento é inesperado, pois imaginava-se que conforme a alocação de memória fosse aumentando, maior seria o impacto do carregamento da função durante o coldstart. 


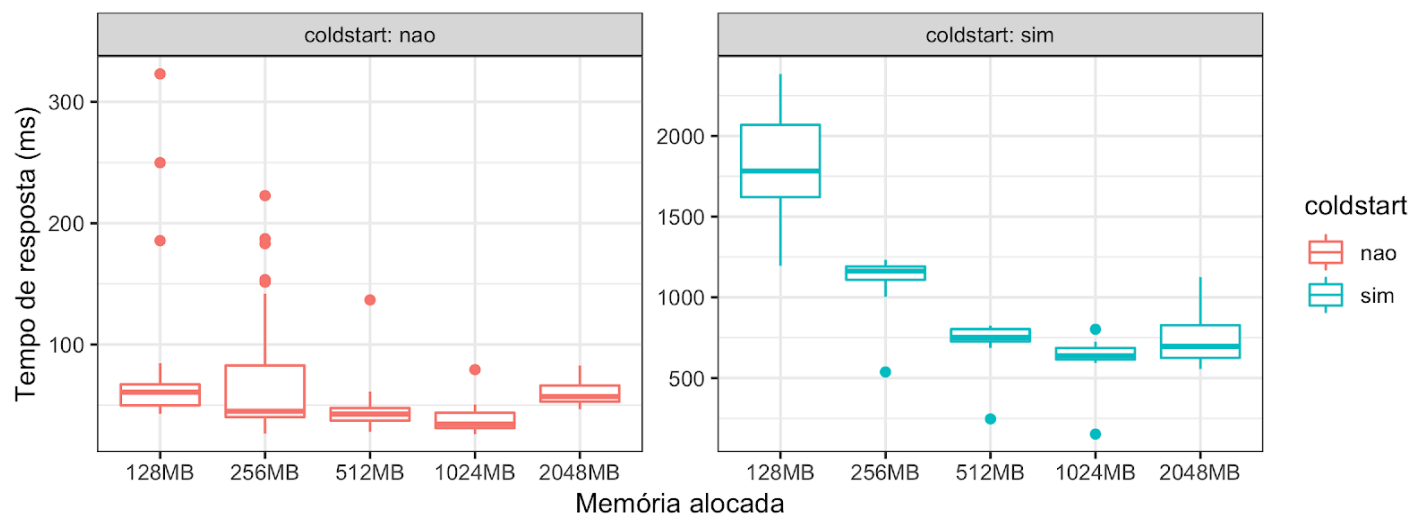

Figura 4. Boxplot do tempo de resposta com e sem coldstart, para diferentes cenários de memória alocada para a função.

Este fenômeno pode ser explicado através da documentação do AWS Lambda, que diz que a capacidade de CPU alocada para a função é proporcional à quantidade de memória alocada para a mesma. Portanto, uma hipótese é que funções que alocam mais memória, como também possuem maior capacidade de CPU, carregam a função e respondem às requisições mais rapidamente. Uma ressalva é que ao aumentar a memória alocada de $1024 \mathrm{MB}$ para $2048 \mathrm{MB}$, não se observou uma diminuição no tempo de resposta; houve, na verdade, um pequeno acréscimo na mediana. Uma possível explicação, no entanto, é que a partir deste ponto, CPU deixa de ser um gargalo e não melhorar mais o desempenho do carregamento e execução da função, enquanto a quantidade maior de memória a ser alocada passa a fazer algum efeito no tempo de carregamento, mesmo que pouco significativo. Além disso, quando não há coldstart (gráfico há esquerda), o tempo de resposta apresenta mais outliers com valores maiores, mostrando que a capacidade menor de CPU também pode afetar o tempo de resposta da função mesmo sem coldstart.

\section{Conclusão}

O modelo de serverless computing está se tornando bastante atrativo para usuários de nuvem, devido à menor complexidade operacional por não precisar gerenciar servidores; da possível redução de custos ao ser cobrado apenas pelo uso da aplicação. Porém, ao desativar funções após um período de ociosidade para reduzir custos, o provedor gera um novo problema para a aplicação chamado coldstart, que é o tempo necessário para inicializar e implantar uma aplicação inativa ao chegar uma nova requisição. Neste trabalho, analisamos o coldstart em um provedor de serverless computing, medindo para diferentes cenários de memória alocada para a função: o tempo que uma função passa ociosa até que o provedor a torne inativa (havendo coldstart); o tempo de resposta das requisições com e sem coldstart; e o overhead que o coldstart impõe no tempo de resposta dependendo da quantidade de memória alocada.

Os resultados dos experimentos mostraram que: (1) Coldstarts aconteceram de forma sistemática após 30 a 42 minutos de ociosidade, dependendo da memória alocada para a função. Alguns coldstarts isolados também ocorrem com tempos de ociosidade menor, de 25 minutos, principalmente para quantidades maiores de memória alocada, o que supõe que em momentos de sobrecarga o provedor pode desativar funções que ocupam mais recursos mais cedo. (2) O overhead imposto pelo coldstart foi significativo para os experimentos realizados, sendo a diferença nas medianas do tempo de resposta com e sem coldstart de aproximadamente $751 \mathrm{~ms}$. (3) Quanto maior a

${ }^{4}$ https://docs.aws.amazon.com/lambda/latest/dg/resource-model.html 
memória alocada, menor tende a ser o overhead do coldstart. A hipótese é que como o provedor aloca CPU proporcionalmente à memória, a função é inicializada e executada mais rapidamente quando há mais memória. Porém, quando se aumentou a memória de $1024 \mathrm{MB}$ para $2048 \mathrm{MB}$, o overhead não diminuiu. A possível explicação é que a CPU deixou de ser um gargalo e aumentar sua capacidade já não afeta o tempo de resposta significativamente.

Com base nos resultados obtidos no experimento, sugerimos que: aplicações que passam muito tempo ociosas podem reduzir custos por não serem cobradas nestes períodos. Porém, se estes períodos forem superiores a 25 minutos e a aplicação for sensível à latência, precisando de tempos de resposta inferiores a 1 segundo para uma grande porcentagem das suas requisições, o overhead imposto pelo coldstart pode afetar a qualidade de serviço da aplicação, sendo mais adequado um serviço tradicional baseado em máquinas virtuais ou containers, ou estratégias para que funções não sejam desativadas. Por outro lado, se as aplicações se mantém ativas e raramente apresentam longos períodos de atividade, a facilidade de uso do modelo de serverless computing pode ser um grande atrativo para os usuários executarem e escalarem suas aplicações sem precisar gerenciar servidores.

Como possíveis trabalhos futuros, pretende-se investigar o impacto no coldstart adotando diferentes provedores de serviços, diferentes linguagens de programação e com experimentos mais exaustivos em dias da semana e horas diferentes para eliminar efeitos externos da análise.

\section{Referencias}

Baldini, I., Castro, P., Chang, K., Cheng, P., Fink, S., Ishakian, V., Mitchell, N., Muthusamy, V., Rabbah, R, Slominski, A. and Suter, P. (2017) "Serverless Computing: Current Trends and Open Problems". In: Research Advances in Cloud Computing. Edited by Chaudhary S., Somani G. and Buyya R, Springer, Singapore.

Cui, Y. "Finding coldstarts: how long does AWS Lambda keep your idle functions around?" (2017a) in: https://theburningmonk.com/2017/06/finding-coldstarts-howlong-does-aws-lambda-keep-your-idle-functions-around/. Acesso em: 28 set. 2018.

Cui, Y "How does language, memory and package size affect cold starts of AWS Lambda?" (2017b) in: https://read.acloud.guru/does-coding-language-memory-orpackage-size-affect-coldstarts-of-aws-lambda-a15e26d12c76/. Acesso em: 22 out. 2018

Fowler, M. "Serverless Architectures" (2018). In: martinfowler.com. Disponível em: https://martinfowler.com/articles/serverless.html. Acesso em: 14 ago. 2018.

Gancarz, R. "Serverless Still Requires Infrastructure Management" (2018). In: InfoQ. Disponível em: https://www.infoq.com/articles/serverless-infrastructuremanagement. Acesso em: 14 ago. 2018.

Ken, F. "Why The Future Of Software And Apps Is Serverless" (2012). In: readwrite. Disponível em: https://readwrite.com/2012/10/15/why-the-future-of-software-andapps-is-serverless. Acesso em: 08 ago. 2018.

Mell, P., Grance, T. (2011). The NIST definition of cloud computing.

McGrath, G., Brenner, P.R. Serverless Computing: Design, Implementation, and Performance, IEEE 37th International Conference on Distributed Computing Systems Workshops, 2017

Perez, C. "Serverless e AWS Lambda" (2018). In: Elo7 Tech. Disponível em: https://engenharia.elo7.com.br/serverless/. Acesso em: 20 ago. 2018.

Savage, N. (2018). Going serverless. Communications of the ACM, 61(2), 15-16. 